\title{
A new device to treat intra-capsular fracture neck of femur non-union
}

\author{
P. Mukherjee • M. J. Ashworth
}

Received: 16 April 2009/Accepted: 13 September 2010/Published online: 29 September 2010

(C) The Author(s) 2010. This article is published with open access at Springerlink.com

\begin{abstract}
In adolescents and young adults, femoral neck fractures often result from high-velocity trauma. These fractures are usually of vertical shear pattern. There is an increased incidence of avascular necrosis and non-union, which is difficult to treat. Non-union of fractured neck of femur in young adults is a serious problem. There is growing evidence that these fractures should be treated with an angle-stable device to improve biomechanics at the fracture site. An ideal implant should prevent varus deformation and retroversion of the fracture in order to prevent failure of the osteosynthesis and thus preventing cut-out of implant and non-union at the fracture site. We report the first use of an Orthofix Gottfried Percutaneous Compression Plate (PC.C.P.) (Orthofix, Guilford, UK) to treat a non-union of an intra-capsular fractured neck of femur. We recommend this, in combination with autologous bone grafting, via a mini hip modification of the Smith-Petersen approach.
\end{abstract}

Keywords Femoral neck fracture · Non-union ·

Dynamic hip screw · Gottfried percuteneous

compression plate $\cdot$ Vertical shear fracture

This study was carried out at Torbay Hospital, Torquay, Devon, UK, TQ27AA.

P. Mukherjee ( $\square)$

Orthopaedic Surgery, Rotherham General Hospital,

Rotherham S60 2UD, UK

e-mail: pavelmukherjee@lycos.com

M. J. Ashworth

Trauma and Orthopaedics, Torbay Hospital, Torquay,

Devon TQ2 7AA, UK

\section{Case report}

A healthy man (aged 17) sustained an isolated displaced intra-capsular fractured neck of the right femur (with posteromedial comminution) in a road traffic accident (Fig. 1). Following initial resuscitation, he underwent closed reduction and internal fixation of the fracture with three cannulated screws (Fig. 2). He was not permitted to weight bear for 6 weeks but then progressed gradually to full weight bearing at 3 months.

He continued to complain of pain in the injured hip. At 5 months, the X-rays showed a persistent fracture gap and the cannulated screws bending. A diagnosis of delayed union was made and the patient referred to this unit. A bone scan revealed the femoral head viable. The inflammatory indices were normal, and a CT scan confirmed the absence of any attempts at healing (Fig. 3). We elected to re-operate because of a lack of evidence of healing and loss of satisfactory alignment from early failure (bending) of the cannulated screws.

At surgery, the screws were extracted. A modified SmithPetersen approach was used to expose and explore the fracture site. Fibrous tissues in the fracture gap and devitalised bone fragments were removed (subsequent microbiological analysis was negative for infection). The bony edges were freshened, and backflow bleeding from femoral head confirmed the head to be viable. Inter-fragmentary autologous bone grafting was performed. This was followed by compression across the fracture gap using a large reduction clamp initially. Definitive stabilisation was accomplished using a Gotfried Percutaneous Compression Plate (PC.C.P, Orthofix SRL, Verona, Italy) inserted through a standard mini-lateral incision and locked in place. The final intraoperative check X-rays were satisfactory (Figs 4, 5).

Post-operatively, the patient was instructed to remain non-weight bearing for 6 weeks followed by a gradual 


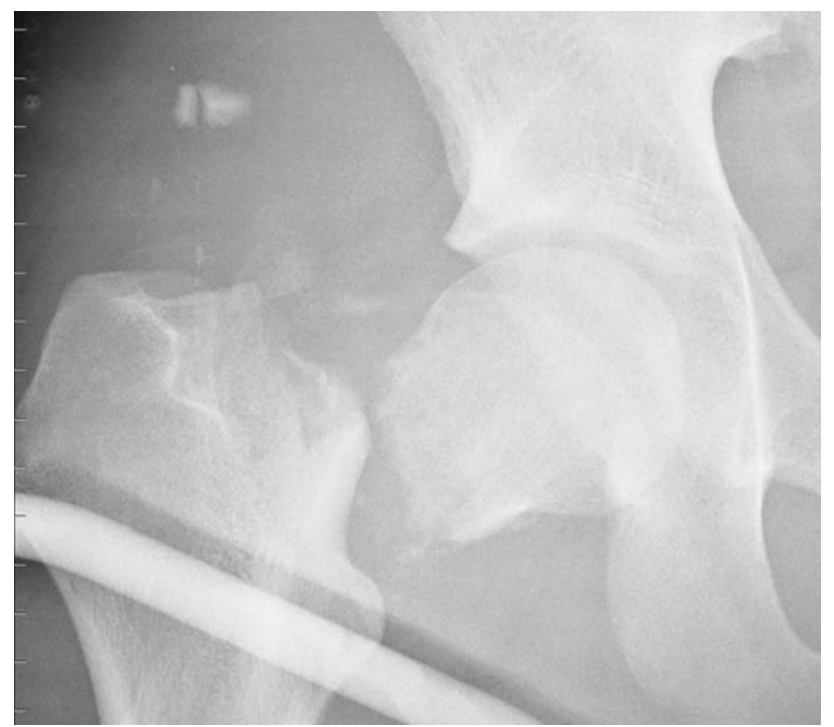

Fig. 1 X-ray showing vertical shear intra-capsular fractured neck of femur with comminution (AP view)

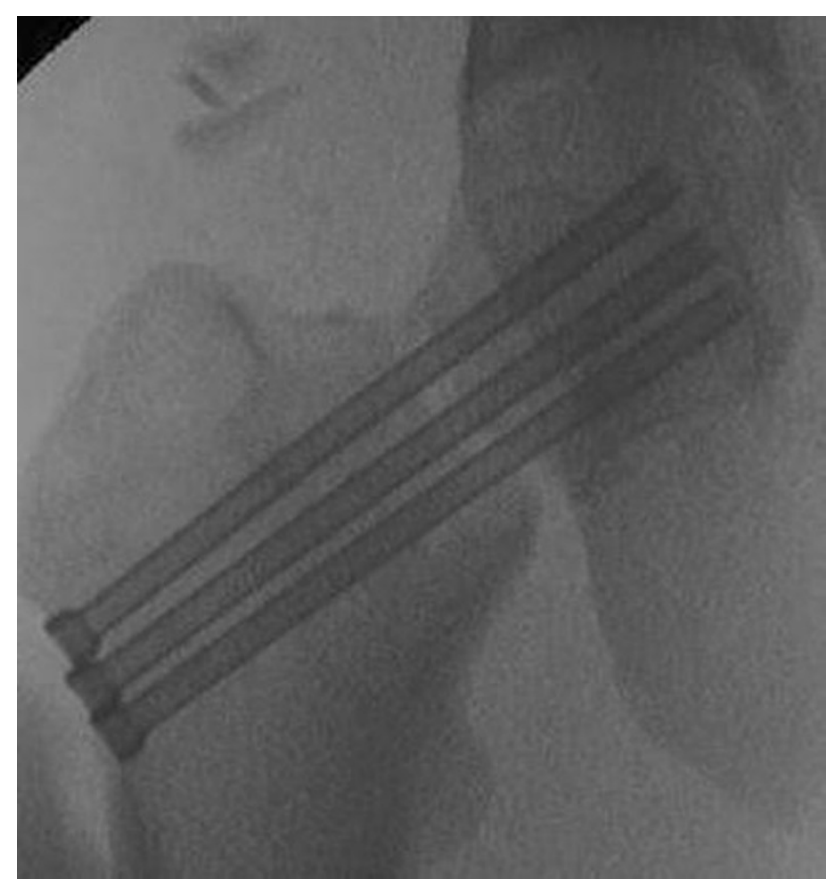

Fig. 2 X-ray showing fracture fixation with three cannulated screws (AP view)

increase to full weight bearing by 3 months. At 6 months, he was fully weight bearing and completely pain-free. A repeat CT scan confirmed bony union (Fig. 6).

\section{Discussion}

A non-union of an intra-capsular fracture of the femoral is difficult to treat in a young adult [1-5]. The principles of

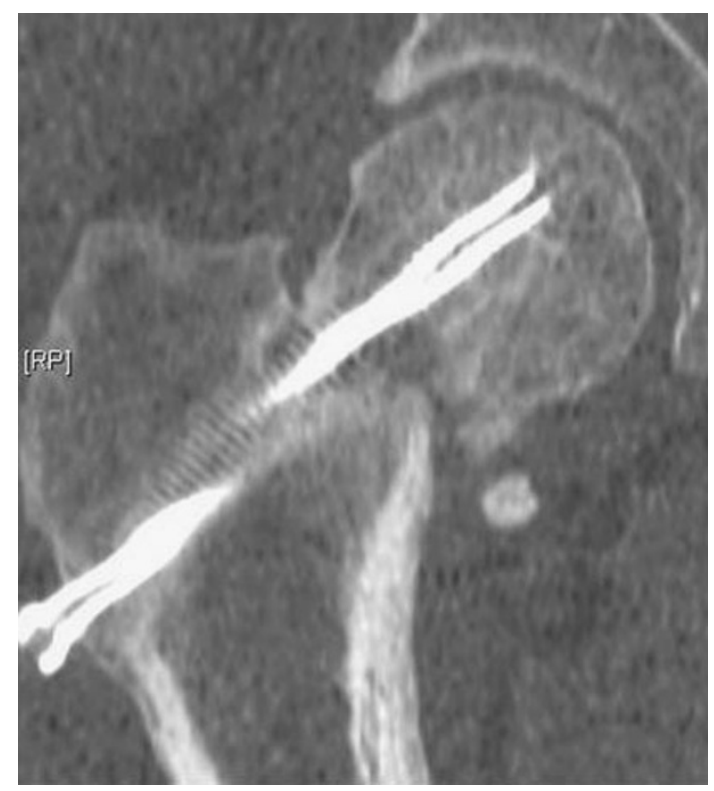

Fig. 3 CT scan showing non-union at the fracture site (coronal cut)

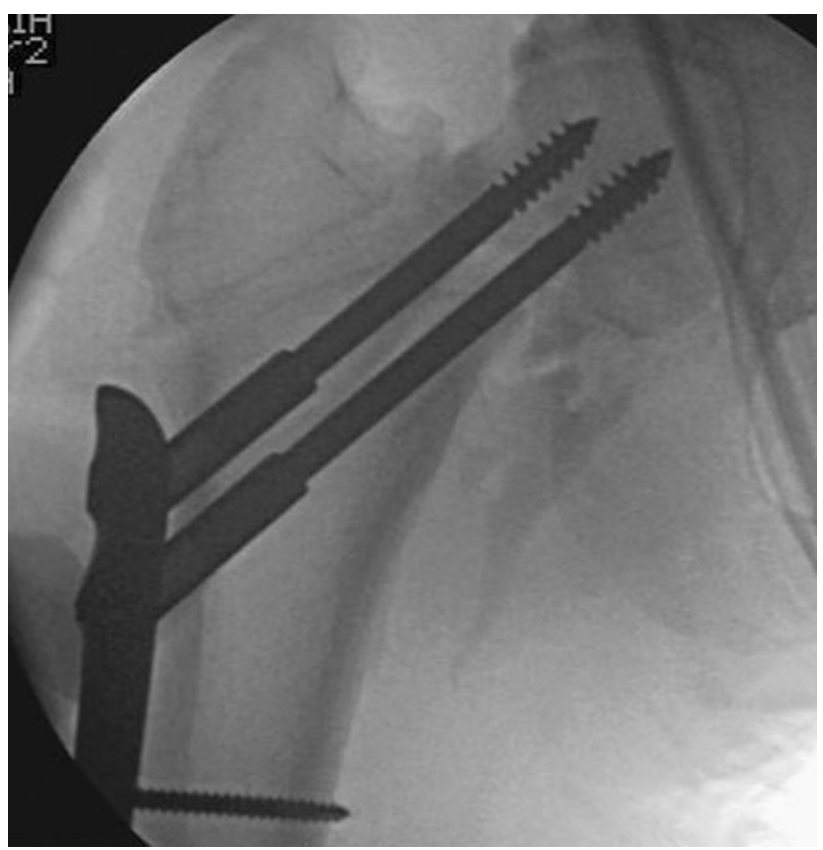

Fig. 4 X-ray sowing PC.C.P. in situ intra-operatively (AP view)

managing non-unions are well established; improve the biology, exclude or treat infection and improve the fracture mechanics. We have used these to good effect:

1. We demonstrated the femoral head was vascularised using technetium bone scanning (this allowed the subsequent application of autologous bone grafting, to stimulate the biological healing process)

2. We excluded infection through preoperative inflammatory indices and by negative microbiology of the operative samples 


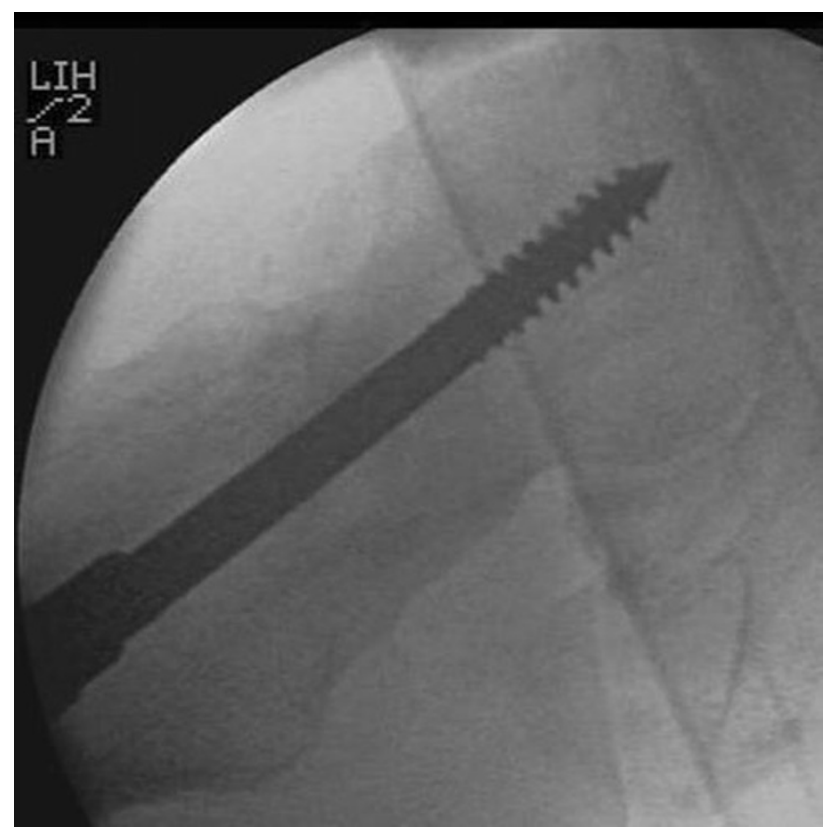

Fig. 5 X-ray showing PC.C.P. in situ intra-operatively (lateral view)

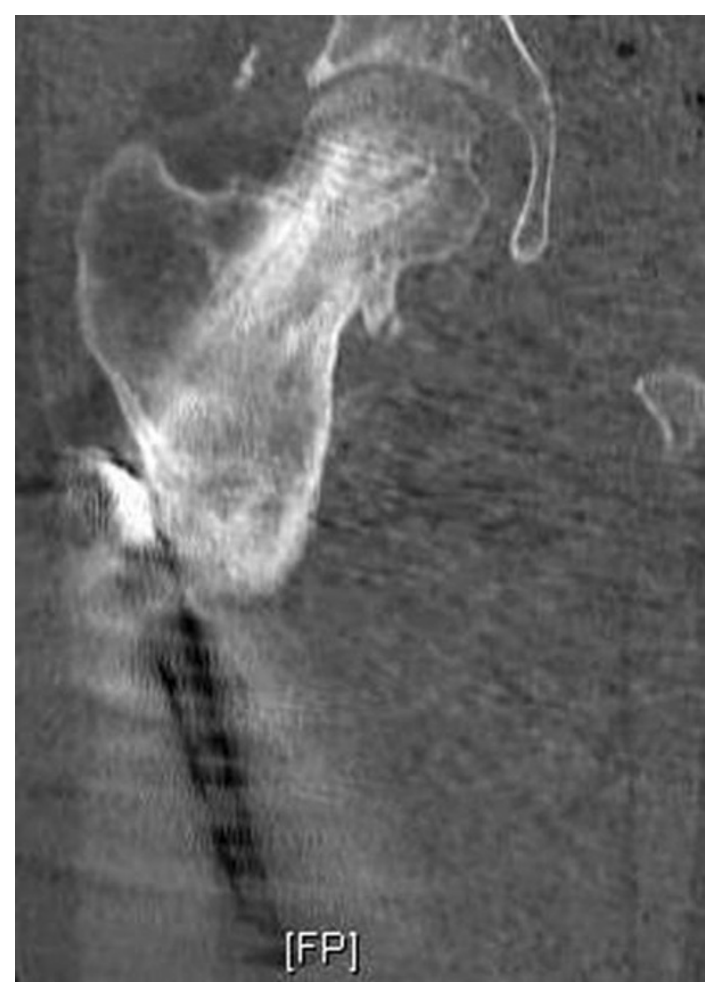

Fig. 6 CT scan showing united fractured neck of femur (coronal cut)

3. We used the PC.C.P. because its improved mechanics allows controlled collapse coupled with rotational stability (this is not provided by the standard dynamic hip screw which has low rotational stability) [6]. The addition of a de-rotation screw to the standard DHS can offset this disadvantage, but this configuration still lacks a stable-angle configuration $[6,7]$

The PC.C.P. is suitable for unstable pertrochanteric femoral neck fractures [8-11]. A recent biomechanical study has shown it to be better than a standard DHS when loaded with combined axial and rotational forces [6]. As such, we believe the PC.C.P. should be considered as a preferred internal fixation device for displaced comminuted intra-capsular femoral neck fractures in young adults. In addition to the choice of an appropriate stabilisation implant, the fracture gap should be visualised so as to allow accurate bony approximation. It is possible that had this been performed at first presentation, a different outcome would have been encountered here. We recommend the use of a modified Smith-Petersen approach [12] to achieve this visualisation because it preserves the blood supply to the femoral head. In this modified approach, instead of creating a plane between the tensor fascia lata (TFL) and the sartorius, the incision is based over the medial substance of the TFL to protect the lateral cuteneous nerve of thigh; the deeper dissection then reflects the TFL laterally to reach the plane between the TFL and sartorius.

\section{Conclusion}

A non-union of an intra-capsular fracture of the neck of femur in a young adult is difficult to treat. The key to the treatment is improving the biology and biomechanical stability at the fracture site. The PC.C.P. offers better rotational and axial stability than a standard DHS. This case describes its successful use in revision surgery. We have used this device successfully in other unusual patterns of femoral neck fractures previously [13] and can recommend its use also for index fracture fixation with due compliance with the biological and biomechanical principles of treatment.

\section{Conflict of interest None.}

Open Access This article is distributed under the terms of the Creative Commons Attribution Noncommercial License which permits any noncommercial use, distribution, and reproduction in any medium, provided the original author(s) and source are credited.

\section{References}

1. Boston DA (1982) Bilateral fractures of the femoral neck. Injury 14:207-210

2. Hirsch C, Frankel VH (1960) Analysis of forces producing fractures of the proximal end of the femur. J Bone Joint Surg Br 42:633-640

3. Gray AJ, Parker MJ (1994) Intra-capsular fractures of the neck of the femur in young patients. Injury 25:667-669 
4. Protzman RF, Burkhalter WE (1976) Femoral neck fractures in young adults. J Bone Joint Surg Am 58:689-695

5. Min B et al (2003) Valgus intertrochanteric osteotomy for non-union of femoral neck fracture. Injury 37:786-790

6. Brandt E et al (2006) Biomechanical analysis of the percutaneous compression plate with sliding hip screw in intracapsular fracture neck of femur Experimental assessment using synthetic and cadaver bone. Injury 37:979-983

7. Swiontkowski M et al (1987) Torsion and bending analysis of internal fixation of femoral neck fractures; the role of implant design and bone density. J Orthop Res 5:433-444

8. Bensafi $\mathrm{H}$ et al (2006) The percutaneous compression plate (PCCP) in the treatment of trochanteric hip fractures in elderly patients. Acta Orthop Belg 72:314-319
9. Brandt SE et al (2002) Percutaneous compression plating (PCCP) versus the dynamic hip screw for pertrochanteric hip fractures: preliminary results. Injury 33:413-418

10. Gotfried Y (2000) Percutaneous compression plating for intertrochanteric hip fractures. J Orthop Trauma 14:490-495

11. Gotfried Y (2002) Percutaneous compression plating for intertrochanteric hip fractures: treatment rationale. Orthopedics 25: 647-652

12. Molnar RB, Routt ML (2007) Open reduction of intracapsular hip fractures using a modified smith-petersen surgical exposure. J Orthop Trauma 21:490-494

13. Poulter RJ, Ashworth MJ (2007) Concomitant ipsilateral subcapital and intertrochanteric fractures of the femur. Injury extra $38: 88-89$ 